\title{
Evaluation of the two-particle propagator for Hubbard model with the help of Hubbard-I approximation
}

\author{
A.V. Rozhkov and A.L. Rakhmanov \\ Institute for Theoretical and Applied Electrodynamics, Russian Academy of Sciences, \\ ul. Izhorskaya, 13, 125412, Moscow, Russia
}

\begin{abstract}
The Hubbard-I approximation is generalized to allow for direct evaluation of the equal-time anomalous two-electron propagator for Hubbard model on twodimensional square lattice. This propagator is compared against the quantum Monte Carlo data obtained by Aimi and Imada [J. Phys. Soc. Jpn. 76, 113708 (2007)] in the limit of strong electron-electron interaction. The Hubbard-I predictions are in a good qualitative agreement with the Monte Carlo results. In particular, $d$-wave correlations decay as $\mathrm{cr}^{-3}$ ("free electron" behaviour), if separation $r$ exceeds 2-3 lattice constants. However, the Hubbard-I approximation underestimates coefficient $c$ by a factor of about three. We conclude that the Hubbard-I approximation, despite its simplicity and artefacts, captures the qualitative behaviour of the two-particle propagator for the Hubbard model, at least for moderate values of $r$.

PACS numbers: $71.27 .+\mathrm{a} 74.20 .-\mathrm{z}$
\end{abstract}




\section{Introduction}

\subsection{General outlook}

Consider the Hubbard model's Hamiltonian [1]:

$$
H=H_{\text {kin }}+H_{\text {int }}-\mu \sum_{\mathbf{i}, \sigma} n_{\mathbf{i} \sigma}
$$

where

$$
H_{\mathrm{kin}}=-t \sum_{\mathbf{i}, \mathbf{a}, \sigma} c_{\mathbf{i} \sigma}^{\dagger} c_{\mathbf{i}+\mathbf{a} \sigma}, \quad H_{\mathrm{int}}=U \sum_{\mathbf{i}} n_{\mathbf{i} \sigma} n_{\mathbf{i} \bar{\sigma}} .
$$

Here $c_{\mathbf{i} \sigma}^{\dagger}$ and $c_{\mathbf{i} \sigma}$ are the creation and annihilation operators for an electron with spin projection $\sigma$ on site $\mathbf{i}$ of the square lattice, $t$ is the hoping integral, $U$ is the on-site Coulomb repulsion, a are vectors connecting the site $\mathbf{i}$ with its nearest neighbors, $\mu$

is chemical potential, $n_{\mathbf{i} \sigma}=c_{\mathbf{i} \sigma}^{\dagger} c_{\mathbf{i} \sigma}$ is the operator of electron number, and $\bar{\sigma}$ means not-sigma.

This Hamiltonian is commonly used to describe systems of strongly correlated electrons. Among these, we mention such important examples as high-Tc superconducting cuprates, manganites, cobaltites, etc. However, there is no commonly accepted method that allows to solve Hubbard model for a generic value of doping.

One of the most intriguing problems of the Hubbard model is the question if the model captures the essential physics of the high-temperature copper-oxide superconductors. At low interaction one expects that the model exhibits Kohn-Luttinger superconductivity [2, 3, 4, 5]. Unfortunately, the critical temperature in such a regime is very small. Thus, it is necessary to study the Hubbard model at moderate or large interaction strengths. Such a regime is tractable only when the concentration of electrons is low [6, 7, 8]. However, the electron concentration for all known copperoxide superconductors is close to one electron per unit cell. Little rigorous knowledge is available for this limit. Different approaches return quite controversial answers: some Monte Carlo (MC) studies argue against superconductivity [9], or against high- $T_{c}$ superconductivity [10] in Hubbard model, other numerical works favor existence of the superconducting ground state with high condensation energy [11].

In the moderate and strong coupling limits there is no guidance from analytical studies as well: in this parameter region the majority of theoretical methods become unreliable. Thus, one is forced to utilize uncontrollable devices with unknown accuracy.

In such a situation it appears useful to investigate reliability of uncontrollable schemes. A particularly convenient uncontrollable approach for the analysis of the Hubbard model is Hubbard-I approximation [1]. Hubbard-I, being invalid for $U \lesssim z t$ (where $z$ is the number of the nearest neighbor sites on the lattice), could be considered as a good first approximation in the case of the strong correlations $U \gg z t$. Its evident advantage is its simplicity. The method allows deriving analytical results in many cases or performing numerical calculations at low computational cost. 
The clarity and transparency of the Hubbard-I approximation explains its popularity among the researchers working in the field of strongly correlated electronic systems. For us, it is especially important that, after the discovery of high-Tc superconductors, the method has been applied to the superconductivity as well. Namely, Hubbard-I approximation is used for study of superconductivity in the Hubbard or extended Hubbard model [12, 13], in the Hubbard model with phonons [14, 15, 16], in the Hubbard model with attractive interaction [17, 18]. A related approach, the two-pole approximation (which, in some respects, is superior to Hubbard-I approximation), and its generalization are applied to study possible superconductivity of the usual Hubbard model [19] and of the multi-band Hubbard model [20]. Similar techniques are used in [21, 22]. Systematic expansion in orders of $t / U$ is developed in [23]. It contains the Hubbard-I approximation as a special case.

\subsection{Our results}

Despite broad use of the Hubbard-I approximation, its ability to account for superconducting properties is untested. In this paper we address this issue by comparing the Cooper pair propagator found with the help of generalized Hubbard-I method against the same propagator calculated within the MC framework (in this paper we refer to our calculations of the two-particle Green's function as the generalized HubbardI approximation to distinguish it from the classical Hubbard-I scheme [1] designed for evaluation of the single-particle Green's function).

Such comparison has became possible only recently due to significant progress of computational techniques [10, 24]. We use the MC data of Aimi and Imada [10], who analyze whether the Hubbard model is enough to capture the phenomenon of high-Tc superconductivity. These authors use an advanced numerical approach (pre-projected Gaussian-basis Monte Carlo). It evaluates the equal-time two-particle propagator in $d_{x^{2}-y^{2}}$-channel $P_{d}(r)$ without any prior assumptions about the structure of the ground state wave function. The MC propagator on 10x10 square lattice does not exhibit superconducting correlations up to $U=7 t$. Instead, when $r$ grows, $P_{d}(r)$ decays algebraically until finite size effects set in.

On our part, we generalize the classic Hubbard-I scheme [1] and derive the equation of motion for the Cooper pair propagator. We determine $P_{d}$ for the doped Hubbard model (1). It is shown that at large $U / t$ the results predicted by the Hubbard-I approach are qualitatively consistent with numerical data obtained in Ref. [10]. In particular,

both approaches agree that the $d_{x^{2}-y^{2}}$-wave pairing correlations decay as $c r^{-3}$ ("free electron" behaviour). The Hubbard-I approximation underestimates coefficient $c$ by a factor of order three. Our findings may be viewed as an attempt to establish the limits of applicability of the Hubbard-I scheme.

The paper is organized as follows. In Sect. 2 we derive the equation of motion for the two-particle propagator. Numerically calculated Hubbard-I correlation function is presented and compared against the Monte Carlo data in Sect. 3. The results obtained 
are discussed in Sect. 4.

\section{Hubbard-I approximation}

\subsection{General idea}

The idea of the Hubbard-I approximation for Hamiltonian (11) is as follows. First, we introduce single-electron Matsubara Green's function $G_{\sigma}(\mathbf{j}-\mathbf{i}, \tau)=-\left\langle\hat{T} c_{\mathbf{j} \sigma}(\tau) c_{\mathbf{i} \sigma}^{\dagger}(0)\right\rangle$, where $\langle\ldots\rangle$ means thermodynamic average, $\hat{T}$ is the time-ordering operator, and $\tau$ is imaginary time. The equation of motion for $G_{\sigma}(\mathbf{j}-\mathbf{i}, \tau)$ can be written as

$$
\begin{aligned}
& \left(-\frac{\partial}{\partial \tau}+\mu\right) G_{\sigma}(\mathbf{j}-\mathbf{i}, \tau)=\delta_{\mathbf{j i}} \delta(\tau)+U F_{\sigma \bar{\sigma}}(\mathbf{j}-\mathbf{i}, \tau) \\
& -t \sum_{\mathbf{a}} G_{\sigma}(\mathbf{j}-\mathbf{i}+\mathbf{a}, \tau)
\end{aligned}
$$

where $\delta_{\mathbf{i j}}$ is the Kronecker symbol, $\delta(\tau)$ is the delta-function, and $F_{\sigma \bar{\sigma}}(\mathbf{j}-\mathbf{i}, \tau)$ is the two-particle Green's function of the form

$$
F_{\sigma \bar{\sigma}}(\mathbf{j}-\mathbf{i}, \tau)=-\left\langle\hat{T} c_{\mathbf{j} \sigma}(\tau) n_{\mathbf{j} \bar{\sigma}}(\tau) c_{\mathbf{i} \sigma}^{\dagger}(0)\right\rangle
$$

Second, we write the equation of motion for function $F$, which includes even more complicated Green's functions, which, in turn, require additional equations of motion, etc. In the Hubbard-I approximation we avoid proliferation of these Green's functions by making the following decoupling:

$$
\begin{aligned}
& \left\langle\hat{T} c_{\mathbf{j}+\mathbf{a} \sigma}(\tau) n_{\mathbf{j} \bar{\sigma}}(\tau) c_{\mathbf{i} \sigma}^{\dagger}(0)\right\rangle \rightarrow\left\langle n_{\mathbf{j} \bar{\sigma}}\right\rangle\left\langle\hat{T} c_{\mathbf{j}+\mathbf{a} \sigma}(\tau) c_{\mathbf{i} \sigma}^{\dagger}(0)\right\rangle, \\
& \left\langle\hat{T} c_{\mathbf{j} \sigma}(\tau) c_{\mathbf{j}+\mathbf{a} \bar{\sigma}}^{\dagger}(\tau) c_{\mathbf{j} \bar{\sigma}}(\tau) c_{\mathbf{i} \sigma}^{\dagger}(0)\right\rangle \rightarrow\left\langle c_{\mathbf{j} \bar{\sigma}} c_{\mathbf{j} \sigma}\right\rangle \\
& \times\left\langle\hat{T} c_{\mathbf{j}+\mathbf{a} \bar{\sigma}}^{\dagger}(\tau) c_{\mathbf{i} \sigma}^{\dagger}(0)\right\rangle=0 \\
& \left\langle\hat{T} c_{\mathbf{j} \sigma}(\tau) c_{\mathbf{j} \bar{\sigma}}^{\dagger}(\tau) c_{\mathbf{j}+\mathbf{a} \bar{\sigma}}(\tau) c_{\mathbf{i} \sigma}^{\dagger}(0)\right\rangle \rightarrow\left\langle c_{\mathbf{j} \sigma} c_{\mathbf{j} \bar{\sigma}}^{\dagger}\right\rangle \\
& \times\left\langle\hat{T} c_{\mathbf{j}+\mathbf{a} \bar{\sigma}}(\tau) c_{\mathbf{i} \sigma}^{\dagger}(0)\right\rangle=0 .
\end{aligned}
$$

To understand the nature of this approximation let us express the electron density as a sum of its average value and fluctuations around it:

$$
\begin{aligned}
& n_{\mathbf{j} \bar{\sigma}}=\left\langle n_{\mathbf{j} \bar{\sigma}}\right\rangle+\delta n_{\mathbf{j} \bar{\sigma}}, \text { where } \\
& \delta n_{\mathbf{j} \bar{\sigma}}=n_{\mathbf{j} \bar{\sigma}}-\left\langle n_{\mathbf{j} \bar{\sigma}}\right\rangle .
\end{aligned}
$$

Two other boson-like quantities, $c_{\mathbf{j} \bar{\sigma}} c_{\mathbf{j} \sigma}$ and $c_{\mathbf{j} \sigma} c_{\mathbf{j} \sigma}^{\dagger}$, can be represented in the same manner. The above decouplings correspond to the assumption that the propagation of a single electron is unaffected by the fluctuations of these quantities around their average values. This assumption could be proven by either showing that (i) the fluctuation terms were small, or that (ii) there were no correlation between the single-electron motion and the fluctuations. Unfortunately, neither (i) nor (ii) are rigorously established. Because of this the Hubbard-I approximation is uncontrollable approach whose accuracy is unknown. 
Using the decouplings (5, 5 ) we derive the equation for $F$ in the form

$$
\begin{aligned}
& \left(-\frac{\partial}{\partial \tau}+\mu-U\right) F_{\sigma \bar{\sigma}}(\mathbf{j}-\mathbf{i}, \tau) \\
& =\left\langle n_{\mathbf{j} \bar{\sigma}}\right\rangle\left[\delta_{\mathbf{j i}} \delta(\tau)-t \sum_{\mathbf{a}} G_{\sigma}(\mathbf{j}-\mathbf{i}+\mathbf{a}, \tau)\right],
\end{aligned}
$$

This equation, together with (3), constitutes a closed system, sufficient for calculation of functions $G$ and $F$. This is how the single-electron propagator is calculated within the framework of the Hubbard-I approximation.

\subsection{Hubbard-I approximation for the two-particle propagator}

Following Ref. [10], we calculate here the equal-time two-electron correlation functions of the form

$$
P_{d}(\mathbf{r})=\frac{1}{2 N} \sum_{\mathbf{i}=1}^{N}\left\langle\Delta_{d}^{\dagger}(\mathbf{i}) \Delta_{d}(\mathbf{i}+\mathbf{r})+\Delta_{d}(\mathbf{i}) \Delta_{d}^{\dagger}(\mathbf{i}+\mathbf{r})\right\rangle,
$$

where $N$ is the number of sites on the square lattice,

$$
\begin{aligned}
& \Delta_{d}(\mathbf{i})=\frac{1}{\sqrt{2}} \sum_{\mathbf{r}} f_{d}(\mathbf{r})\left(\Delta_{\mathbf{i}+\mathbf{r}}+\Delta_{\mathbf{i}+\mathbf{r i}}\right), \\
& \Delta_{\mathbf{i} \mathbf{j}}=c_{\mathbf{i} \sigma}^{\dagger} c_{\mathbf{j} \bar{\sigma}}^{\dagger} .
\end{aligned}
$$

The form-factor

$$
f_{d}(\mathbf{r})=\delta_{r_{y}, 0}\left(\delta_{r_{x}, 1}+\delta_{r_{x},-1}\right)-\delta_{r_{x}, 0}\left(\delta_{r_{y}, 1}+\delta_{r_{y},-1}\right)
$$

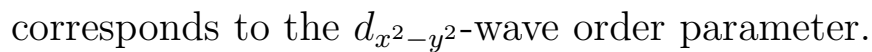

To calculate $P_{d}(\mathbf{r})$ let us first define the propagator

$$
P_{\mathrm{ijlm}}(\tau)=\left\langle\hat{T} \Delta_{\mathbf{i j}}(\tau) \Delta_{\mathrm{lm}}^{\dagger}(0)\right\rangle .
$$

The equal-time two-electron correlation function $P_{d}(\mathbf{r})$ is related to $P_{\mathrm{ijlm}}(\tau)$ as

$$
\begin{aligned}
& P_{d}(\mathbf{r})=\frac{1}{4 N} \sum_{\mathbf{i} \mathbf{b}^{\prime}} f_{d}(\mathbf{b}) f_{d}\left(\mathbf{b}^{\prime}\right)\left[P_{\mathbf{i}, \mathbf{i}+\mathbf{b}, \mathbf{i}+\mathbf{r}, \mathbf{i}+\mathbf{b}^{\prime}+\mathbf{r}}(\tau)\right. \\
& \left.+P_{\mathbf{i}+\mathbf{b}, \mathbf{i}, \mathbf{i}+\mathbf{r}, \mathbf{i}+\mathbf{b}^{\prime}+\mathbf{r}}(\tau)+P_{\mathbf{i}, \mathbf{i}+\mathbf{b}, \mathbf{i}+\mathbf{b}^{\prime}+\mathbf{r}, \mathbf{i}+\mathbf{r}}(\tau)\right] \\
& \left.+P_{\mathbf{i}+\mathbf{b}, \mathbf{i}, \mathbf{i}+\mathbf{b}^{\prime}+\mathbf{r}, \mathbf{i}+\mathbf{r}}(\tau)\right]\left.\right|_{\tau \rightarrow-0}+(\tau \rightarrow+0, \mathbf{r} \rightarrow-\mathbf{r}) .
\end{aligned}
$$

We write down equation of motion for the propagator $P_{\mathrm{ijlm}}$ using Hamiltonian (1) and (2)

$$
\begin{aligned}
& \left(\frac{\partial}{\partial \tau}+2 \mu\right) P_{\mathbf{i j l m}}=-t \sum_{\mathbf{a}}\left(P_{\mathbf{i}+\mathbf{a j l m}}+P_{\mathbf{i j}+\mathbf{a l m}}\right) \\
& +\delta(\tau)\left(\left\langle c_{\mathbf{i} \sigma}^{\dagger} c_{\mathbf{l} \sigma}\right\rangle \delta_{\mathbf{j} \mathbf{m}}-\left\langle c_{\mathbf{m} \bar{\sigma}} c_{\mathbf{j} \bar{\sigma}}^{\dagger}\right\rangle \delta_{\mathbf{i l}}\right)+U \mathcal{P}_{\mathbf{i j} \mathbf{l} \mathbf{m}},
\end{aligned}
$$

where the three-particle propagator is defined as

$$
\mathcal{P}_{\mathbf{i j} \mathbf{l m}}(\tau)=\left\langle\hat{T}\left[\Delta_{\mathbf{i j}}(\tau) n_{\mathbf{j} \sigma}(\tau)+n_{\mathbf{i} \bar{\sigma}}(\tau) \Delta_{\mathbf{i j}}(\tau)\right] \Delta_{\mathbf{l m}}^{\dagger}(0)\right\rangle .
$$


The double occupancy is very unlikely: $P_{\mathbf{i j l m}}=o(t / U) \approx 0$ if $\mathbf{i}=\mathbf{j}$ or $\mathbf{l}=\mathbf{m}$. Assuming the absence of the magnetic order, which means, in particular, $\left\langle c_{\mathbf{i} \sigma} c_{\mathbf{j} \sigma}^{\dagger}\right\rangle=\left\langle c_{\mathbf{i} \bar{\sigma}} c_{\mathbf{j} \sigma}^{\dagger}\right\rangle$, we transform (16) in the Hubbard-I approximation to

$$
\begin{aligned}
& \left(\frac{\partial}{\partial \tau}+2 \mu\right) P_{\mathbf{i j l m}}=-t \sum_{\mathbf{a}}\left(P_{\mathbf{i}+\mathbf{a j l m}}+P_{\mathbf{i j}-\mathbf{a l m}}\right)\left(1-\delta_{\mathbf{i}+\mathbf{a j}}\right) \\
+ & \delta(\tau)\left(\left\langle c_{\mathbf{i} \sigma}^{\dagger} c_{\mathbf{l} \sigma}\right\rangle \delta_{\mathbf{j} \mathbf{m}}-\left\langle c_{\mathbf{m} \sigma} c_{\mathbf{j} \sigma}^{\dagger}\right\rangle \delta_{\mathbf{i l}}\right)+U \mathcal{P}_{\mathbf{i j l m}},
\end{aligned}
$$

which is valid if $\mathbf{i} \neq \mathbf{j}$ and $\mathbf{l} \neq \mathbf{m}$.

Now we need to derive an equation for $\mathcal{P}$. The required calculations are onerous, but straightforward. They are presented in Appendix A. Here we quote the final result:

$$
\begin{aligned}
& U \mathcal{P}_{\mathbf{i j l m}} \approx t\left\langle n_{\mathbf{i} \sigma}\right\rangle \sum_{\mathbf{a}}\left(P_{\mathbf{i}+\mathbf{a j l m}}+P_{\mathbf{i j}-\mathbf{a l m}}\right)\left(1-\delta_{\mathbf{i}+\mathbf{a j}}\right) \\
& -\delta(\tau)\left\langle n_{\mathbf{i} \sigma}\right\rangle\left(\left\langle c_{\mathbf{i} \sigma}^{\dagger} c_{\mathbf{l} \sigma}\right\rangle \delta_{\mathbf{j} \mathbf{m}}-\left\langle c_{\mathbf{m} \sigma} c_{\mathbf{j} \sigma}^{\dagger}\right\rangle \delta_{\mathbf{i l}}\right) .
\end{aligned}
$$

This relation is derived under the assumption that the magnetic order is absent: $\left\langle n_{\mathbf{i} \sigma}\right\rangle=\left\langle n_{\mathbf{i} \bar{\sigma}}\right\rangle$.

Substituting (19) in (18), we obtain the equation of motion for the propagator $P_{\mathbf{i j l m}}$ in the Hubbard-I approximation valid when $\mathbf{i} \neq \mathbf{j}$ and $\mathbf{l} \neq \mathbf{m}$

$$
\begin{aligned}
& \left(\frac{\partial}{\partial \tau}+2 \mu\right) P_{\mathbf{i j l m}}= \\
& -\tilde{t} \sum_{\mathbf{a}}\left[P_{\mathbf{i}+\mathbf{a j l m}}\left(1-\delta_{\mathbf{j} \mathbf{i}+\mathbf{a}}\right)+P_{\mathbf{i j}+\mathbf{a l m}}\left(1-\delta_{\mathbf{i j}+\mathbf{a}}\right)\right] \\
& +\delta(\tau)\left(1-\left\langle n_{\mathbf{i} \sigma}\right\rangle\right)\left(\left\langle c_{\mathbf{i} \sigma}^{\dagger} c_{\mathbf{l} \sigma}\right\rangle \delta_{\mathbf{j} \mathbf{m}}-\left\langle c_{\mathbf{m} \sigma} c_{\mathbf{j} \sigma}^{\dagger}\right\rangle \delta_{\mathbf{i l}}\right) .
\end{aligned}
$$

Here $\tilde{t}=t\left(1-\left\langle n_{\mathbf{i} \sigma}\right\rangle\right)$ if the system is uniform.

As we wrote above, $P_{\mathrm{iilm}} \sim o(t / U) \approx 0$ within our accuracy. Thus, jumps from site $\mathbf{j}=\mathbf{i}+\mathbf{a}$ to site $\mathbf{i}$ must be excluded. The same is true for jumps from $\mathbf{i}=\mathbf{j}+\mathbf{a}$ to $\mathbf{j}$. To take this fact into account and to generalize the last equation for the case $\mathbf{i}=\mathbf{j}$, we add the term $t\left(1-\left\langle n_{\mathbf{i} \sigma}\right\rangle\right) \sum_{\mathbf{a}}\left(\delta_{\mathbf{i}+\mathbf{a j}} P_{\mathbf{i}+\mathbf{a j l m}}+\delta_{\mathbf{i j}+\mathbf{a}} P_{\mathbf{i j}+\mathbf{a l m}}\right)$ to the right-hand side of (20) Therefore:

$$
\begin{aligned}
& \left(\frac{\partial}{\partial \tau}+2 \mu\right) P_{\mathbf{i j l m}}= \\
& -\tilde{t} \sum_{\mathbf{a}}\left[P_{\mathbf{i}+\mathbf{a j l m}}\left(1-\delta_{\mathbf{j} \mathbf{i}+\mathbf{a}}-\delta_{\mathbf{i j}}\right)+P_{\mathbf{i j}+\mathbf{a l m}}\left(1-\delta_{\mathbf{i j}+\mathbf{a}}-\delta_{\mathbf{i j}}\right)\right] \\
& +\delta(\tau)\left(1-\left\langle n_{\mathbf{i} \sigma}\right\rangle\right)\left(\left\langle c_{\mathbf{i} \sigma}^{\dagger} c_{\mathbf{l} \sigma}\right\rangle \delta_{\mathbf{j} \mathbf{m}}-\left\langle c_{\mathbf{m} \sigma} c_{\mathbf{j} \sigma}^{\dagger}\right\rangle \delta_{\mathbf{i l}}\right) .
\end{aligned}
$$

In such equation the quantity $P_{\text {iilm }}$ is decoupled from $P_{\mathbf{i j l m}}, \mathbf{i} \neq \mathbf{j}$, which is equivalent to the condition $P_{\text {iilm }}=0$.

Expression (21) corresponds to propagation of two interacting particles: the terms with the Kronecker symbols may be regarded as an effective repulsive interaction between electrons on neighboring sites with the coupling constant of the order of $t(1-$ $\left.\left\langle n_{\mathbf{i} \sigma}\right\rangle\right)$. Thus, we reduce the strong-coupling many-body problem to the intermediatecoupling two-body one. 


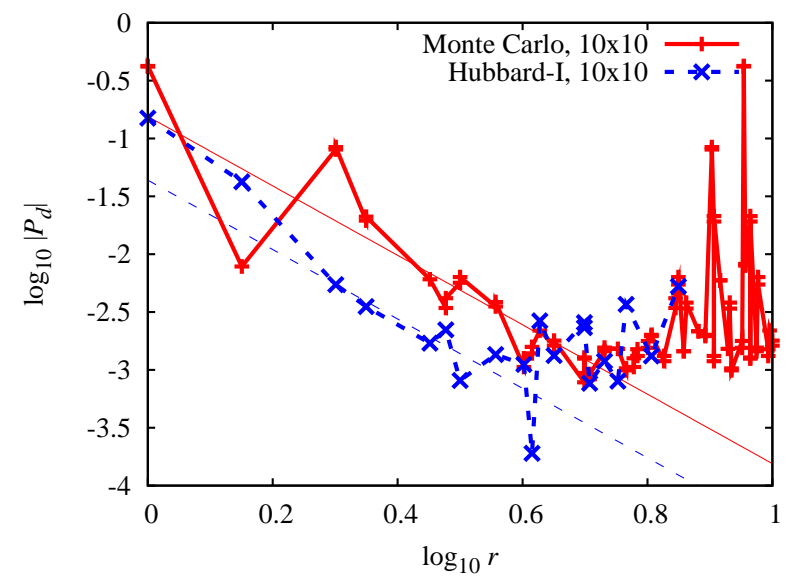

Figure 1. (Colour online) The two-particle propagator $P_{d}(r)$, equation (10). Solid (red) line with right crosses is the Monte Carlo data for the doping level of 0.18 and $U=6$ collected on 10x10 lattice, Ref. [10. Dashed (blue) line with skew crosses is the Hubbard-I result for the doping level of 0.17 on $10 \times 10$ lattice. The two straight lines correspond to $c / r^{3}$ behaviour.

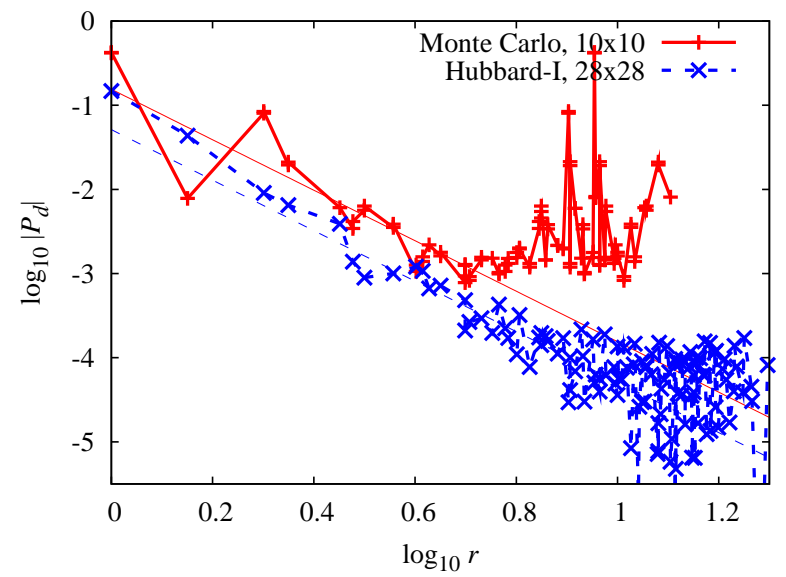

Figure 2. (Colour online) The two-particle propagator $P_{d}(r)$, equation (10). Solid (red) line with right crosses is the Monte Carlo data for the doping level of 0.18 and $U=6$ collected on 10x10 lattice, Ref. [10. Dashed (blue) line with skew crosses is the Hubbard-I result for the doping level of 0.18 on $28 \times 28$ lattice. Two straight lines correspond to $c / r^{3}$ behaviour.

\section{Numerical results}

\subsection{Hubbard-I vs. $M C$}

In this section we present the results of our numerical calculation of the equal-time twoelectron correlation function (10) at a given doping level. The actual calculations are based on Eq. (C.4 derived in Appendix B and Appendix C Doping is equal to $1-n$, where the average number of electrons per site is defined as $n=\sum_{\sigma}\left\langle n_{\mathbf{i} \sigma}\right\rangle$. The average 


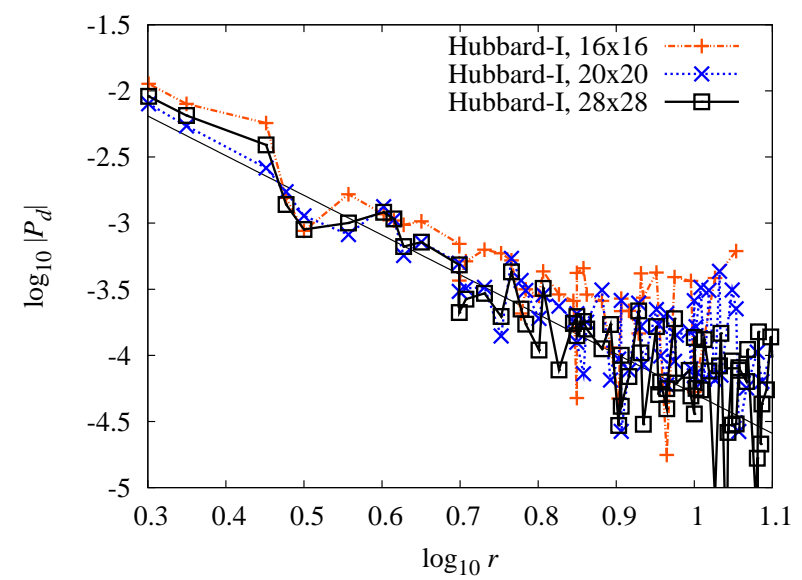

Figure 3. (Colour online) Finite-size scaling for the Hubbard-I propagator: the data for the lattices of three different sizes $(16 \times 16,20 \times 20$, and 28x28) and the doping levels close to 0.18 are plotted. While the curve for the smallest lattice (brown curve with right crosses) deviates substantially from the remaining two, the latter are quite close to each other for sufficiently small separations $\left(\log _{10} r<0.8\right)$.

$\left\langle n_{\mathbf{i} \sigma}\right\rangle$ within the Hubbard-I approximation is

$$
\begin{aligned}
\left\langle n_{\mathbf{i} \sigma}\right\rangle & =\left(1-\left\langle n_{\mathbf{i} \sigma}\right\rangle\right) n_{0}, \\
n_{0}(\mu) & =\frac{1}{N} \sum_{\mathbf{k}} n_{\mathrm{F}}\left(\tilde{\varepsilon}_{\mathbf{k}}-\mu\right) .
\end{aligned}
$$

From this equation the expression for $n$ is found:

$$
n=\frac{2 n_{0}(\mu)}{1+n_{0}(\mu)} .
$$

Therefore, the doping level can be adjusted by changing $\mu$.

For calculation at zero temperature it is convenient to use the substitution in ([C.4):

$$
N_{\mathrm{B}}(\varepsilon)=\frac{1}{\operatorname{sh}(\varepsilon / T)}-n_{\mathrm{F}}(\varepsilon) .
$$

At $T=0$ the Fermi distribution function is zero (unity) for $\varepsilon>0(\varepsilon<0)$. Contribution form $1 / \operatorname{sh}(\varepsilon / T)$ vanishes at small temperature.

Numerical results are shown in figure 1 and figure2, where the propagator is plotted versus separation $r=|\mathbf{r}|$ on the $\log$-log graph. The Hubbard-I propagator $P_{H I}$ is shown as the dashed (blue) line. For comparison, the same propagator computed by T. Aimi and M. Imada [10] using the assumption-free quantum Monte Carlo procedure, $P_{M C}$, is shown by solid (red) line. The subscript 'HI' stands for 'Hubbard-I', the subscript 'MC' - for 'Monte Carlo'.

In figure 1 we present the Hubbard-I data calculated on a 10x10 lattice for the doping level of 0.17 . The same lattice size is used in the MC simulation of Ref. [10]. The MC data is collected for the doping level of 0.18 . The small discrepancy in the densities is unavoidable in our situation. Indeed, due to the finite size of the system, the 
number of particles cannot be changed continuously. In addition, in case of Hubbard-I scheme, the number of physical electrons is not an independent quantity, but rather has to be calculated according to formula (24). Given these two circumstances, it is impossible in general to match the MC and Hubbard-I densities exactly. However, such a small discrepancy (less than 6\%) is of little importance for our goal.

The solid straight line fits the MC propagator in the window $0.32<\log _{10} r<0.78$. Such fit corresponds to $c / r^{3}$ decay, as discussed in Ref. [10]. Propagator $P_{H I}$ is also fited by a straight line (blue dashed straight line) in the window $0.3<\log _{10} r<0.6$.

To extract the asymptotic behaviour of $P_{H I}(r)$ more reliably it is better to look at the propagator on a bigger lattice. In figure 3 it is shown how the propagator converges for large lattices. We observe that the curves for the lattices with $M \geq 20$ almost coincide for moderate $r$. Thus, such systems can be used to find the asymptotic. To that end, let us examine figure 2. It presents the Hubbard-I data for 28x28 lattice is shown together with the MC data. Analysis of figure 2 reveals that the difference between assumption-free MC and much more simple Hubbard-I calculations is not large, moreover, qualitative appearances of the curves are similar. In particular, both curves decay as a power-law $c / r^{\nu}$. In [10] it is established that $\nu \approx 3$, which is consistent with the free-electron behaviour. To determine this exponent for the Hubbard-I correlation function we use two-parameter least-square fitting procedure for the Hubbard-I data in the range $0.3<\log _{10} r<0.9$. The fitting returns the following values:

$$
\log _{10} c_{\mathrm{FIT}}=-1.2 \pm 0.1, \nu_{\mathrm{FIT}}=3.1 \pm 0.2 \text {. }
$$

The value of $\nu_{\text {FIT }}$ is consistent with the MC result. Below we will always assume that the Hubbard-I correlation function decays with $\nu=3$. Deviations from the free-electron behaviour at large $r$ are a manifestation of the finite-size effects.

The fact that the MC propagator decays as the free-electron propagator is a surprising revelation of Ref. [10]. The ability of the Hubbard-I approximation to capture the $1 / r^{3}$ law is a rather simple consequence of the equation of motion, (21). Indeed, in the latter equation, if $r$ is large, the interaction terms (the terms with the Kronecker symbols) contribute to the $s$-wave channel only; as for the $d$-wave propagator, its equation of motion coincides, up to a normalization factor, with that for free fermions.

In figure 2 the obtained values of the propagators are "noisy". These oscillations arise, because, in general, the propagators depend not only on the distance $r$, but on the direction of the vector $\mathbf{r}$ as well. Despite the "noise", one can easily observe that the Hubbard-I propagator $P_{H I}$ is smaller than the Monte Carlo propagator $P_{M C}$.

To examine $P_{H I}$ and $P_{M C}$ in a more rigorous manner let us quantify the $1 / r^{3}$ behaviour of the propagators. For this aim we fit both functions in figure 2 by straight lines in the window $0.32<\log _{10} r<0.78$. The fit lines are defined by the equations:

$$
\begin{aligned}
& \log _{10} P_{M C}=-0.81-3 \log _{10} r, \\
& \log _{10} P_{H I}=-1.29-3 \log _{10} r,
\end{aligned}
$$

which correspond to $P_{d}(r) \approx c / r^{3}$ with $c_{M C} \approx 0.15, c_{H I} \approx 0.051$, and the ratio $c_{M C} / c_{H I} \approx 2.9$. Thus, the Hubbard-I propagator's asymptotic coincides with the 


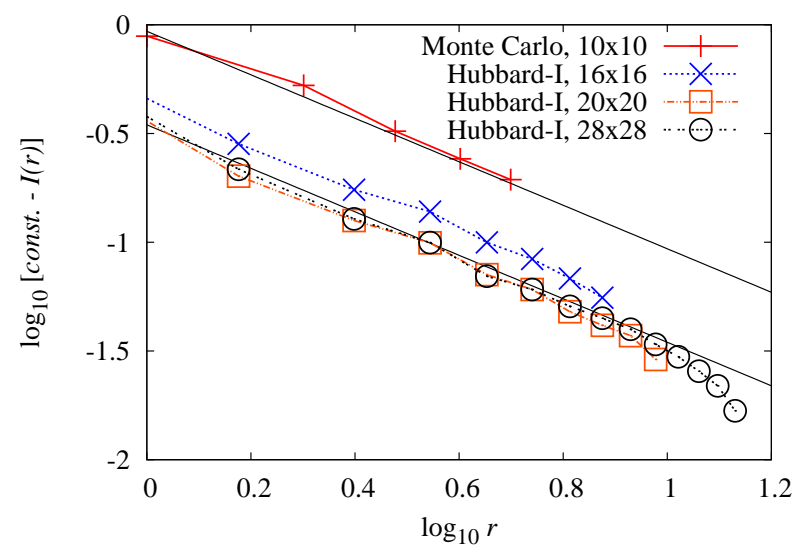

Figure 4. (Colour online) Function $[\alpha-I(r)]$, equation (29); solid (red) line with right crosses is the Monte Carlo data. Three other lines are the Hubbard-I results for different lattice sizes. The parameters are the same as in figure 2 Two straight lines fit the propagators at moderate $r$ according to equation (32). The values of $\alpha$ 's are as follows: $\alpha_{M C}=4.1, \alpha_{H I}^{16}=1.45, \alpha_{H I}^{20} \approx \alpha_{H I}^{28}=1.40$.

asymptotic of the propagator calculated with the help of the assumption-free Monte Carlo method within an order of magnitude, but, of course, not exactly.

We can further compare the Hubbard-I and Monte Carlo data by eliminating "noise" in the propagators with the help of the following procedure. Define the sum

$$
I(r)=\sum_{\sqrt{x^{2}+y^{2}}<r} P_{d}(x, y) .
$$

If we assume that $P_{d}(x, y) \sim c(\phi) r^{-3}$, where $\phi$ is the polar angle, then $I(r)$ can be approximated as

$$
I(r) \approx \int_{x^{2}+y^{2}<r^{2}} d x d y P(x, y) \approx \alpha-\frac{\beta}{r}
$$

where

$$
\beta=\int_{0}^{2 \pi} c(\phi) d \phi=2 \pi \bar{c}
$$

and the constant $\alpha$ can be estimated as $\alpha \approx I\left(r_{\max }\right)$, with $r_{\max }=M / 2$. Function $I(r)$, by its definition, is independent of vector r's direction. Consequently, it is smooth function, free from irregular oscillations present in figure 2. This makes the fitting procedure more robust.

Dependence $\log _{10}(\alpha-I)$ vs. $\log _{10} r$ is plotted in figure 4 for the same values of parameters as the data in figure 2. Similarly to the data presented in figure [3, the 16x16 curve lies somewhat away from the curves corresponding to the larger lattices. The curves for 20x20 and 28x28 lattices coincide with each other almost everywhere, suggesting that for systems of such sizes the finite-size effects are important for large $r$ only. 


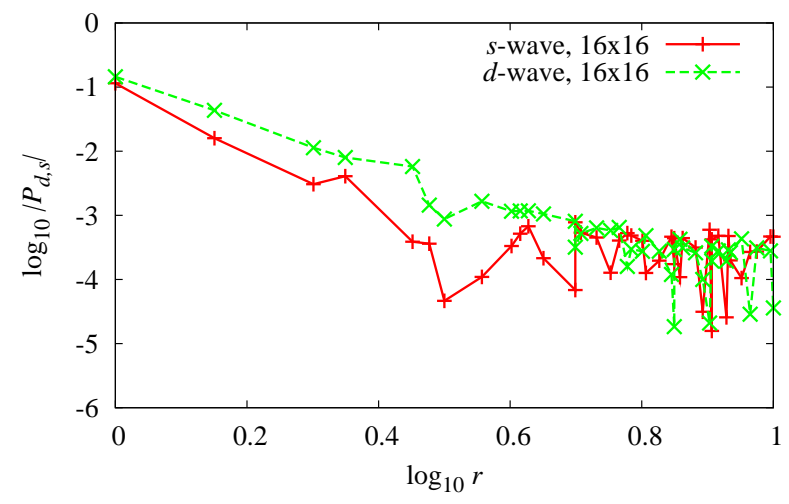

Figure 5. (Colour online) Dependence of $d$-wave and $s$-wave propagators on $r ; P_{d}(r)$ - dashed (green) line and $P_{s}(r)$ - solid (red) line. Both propagators are calculated with the help of Hubbard-I approximation for the doping level of 0.19 on 16x16 lattice.

To determine the asymptotic parameters the curves in figure 4 are fitted by the straight lines:

$$
\log _{10}(\alpha-I)=\log _{10} 2 \pi \bar{c}-\log _{10} r .
$$

The values of the fitting constants are: $\bar{c}_{M C} \approx 0.15, \bar{c}_{H I} \approx 0.055$. As expected, they are close to $c_{M C}$ and $c_{H I}$.

\subsection{Application}

The numerical procedure in the case of the Hubbard-I approximation is much simpler than the quantum Monte Carlo computations. One can use it to derive different properties of the Hamiltonian (2). As an example, we apply the Hubbard-I approximation (B.10) to calculation of the equal-time two-electron correlation function in the $s$-channel:

$$
P_{s}(\mathbf{r})=\frac{1}{2 N} \sum_{\mathbf{i}=1}^{N}\left\langle\Delta_{s}^{\dagger}(\mathbf{i}) \Delta_{s}(\mathbf{i}+\mathbf{r})+\Delta_{s}(\mathbf{i}) \Delta_{s}^{\dagger}(\mathbf{i}+\mathbf{r})\right\rangle,
$$

where

$$
\Delta_{s}(\mathbf{i})=\frac{1}{\sqrt{2}} \sum_{\mathbf{r}} f_{s}(\mathbf{r})\left(\Delta_{\mathbf{i}+\mathbf{r}}+\Delta_{\mathbf{i}+\mathbf{r i}}\right),
$$

and $f_{s}$ is the $s$-wave form-factor

$$
f_{s}(\mathbf{r})=\delta_{r_{y} 0}\left(\delta_{r_{x} 1}+\delta_{r_{x}-1}\right)+\delta_{r_{x} 0}\left(\delta_{r_{y} 1}+\delta_{r_{y}-1}\right) .
$$

The result is shown in figure 5 by solid (red) line. Function $P_{s}(r)$ decays quickly for small $r$. At larger $r$ it oscillates around finite-value plateau. As could be expected, the $s$-wave superconducting correlations are weaker and decay faster than the $d$-wave ones [compare solid (red) and dashed (green) lines in figure [5. This is because the electrons in $s$ channel experience strong repulsion suppressing the superconducting correlations. 
Note also that the Hubbard-I correlation functions show no sign of the superconducting instability in both $s$ and $d$ channels: both correlation functions decay at large $r$, while in a superconducting phase the order parameter correlation function must saturate at large distances.

\section{Discussion}

We calculated two-electron equal-time $d$-wave propagator using the Hubbard-I approximation and compared the results with quantum Monte Carlo computations [10]. The results of both approaches are in good qualitative agreement. Specifically, both methods predict that the correlation function decays as $1 / r^{3}$ at $r$ exceeding several lattice constants. This allows us to assess the reliability of the Hubbard-I approximation.

Technically speaking, the $1 / r^{3}$ decay of the Hubbard-I propagator is a consequence of the fact that the interaction in the equation of motion, (21), is important in the $s$-channel only, whereas in the $d$-channel its effect decreases when $r$ increases.

The Hubbard-I approximation underestimates the value of the propagator residue by a factor of order three. Such discrepancy is rather reasonable for an uncontrollable approach.

Our results for the two-particle correlation functions suggest that the Fermi liquid is a poor approximation for the Hubbard model near half-filling. We show that in $d$-wave channel the correlation function decays as $r^{-3}$, which is consistent with "free fermions". However, fast decay of the $s$-wave propagator (see figure 5) does not agree with the Fermi liquid picture. One, however, must remember that, unlike the $d$-wave propagator, the Hubbard-I $s$-wave correlation function is not compared against a controllable method, thus, its accuracy is unknown.

Interpreting our calculation one should keep in mind the following restriction. Since the MC data for large $r$ is not available, we cannot say how Hubbard-I approximation fairs for $r$ exceeding 5-6 lattice constants. The MC data of Ref. [10] cannot be used to rule out the possibility that the Hubbard model exhibits superconductivity with the correlation length larger than 5-6 lattice constants. Our method in its present form is too crude to capture the superconducting correlations.

To conclude, generalizing the Hubbard-I approximation we derive the equation of motion for the two-particle propagator. For the square lattice of finite size this equation is solved numerically. It is demonstrated that the Hubbard-I propagator is consistent with the MC data up to a numerical coefficient of order 3. Thus, Hubbard-I method can be used to approximate the two-particle propagator of the Hubbard model, at least at a qualitative level at moderate separations.

\section{Acknowledgments}

The authors would like to thank Prof. Imada who kindly provided the Monte Carlo data shown in figure 1 and figure 2. Discussions with M.Yu. Kagan are gratefully 
Two-particle propagator with the help of Hubbard-I approximation

acknowledged. The support of RFBR (grants 09-02-00248, 09-02-92114, 08-02-00212) is acknowledged.

\section{Appendix A. Derivation of Eq. (19)}

Here we obtain Eq. (19) assuming that $\mathbf{i} \neq \mathbf{j}$ and $\mathbf{l} \neq \mathbf{m}$. The equation of motion for $\mathcal{P}_{\mathrm{ijlm}}$ is

$$
\dot{\mathcal{P}}_{\mathrm{ijlm}}=\left\langle\hat{T} C(\tau) \Delta_{\mathrm{lm}}^{\dagger}(0)\right\rangle+\delta(\tau)\left\langle\left[\left(n_{\mathbf{i} \bar{\sigma}}+n_{\mathbf{j} \sigma}\right) \Delta_{\mathbf{i j}}, \Delta_{\mathrm{lm}}^{\dagger}\right]\right\rangle,
$$

where we introduced the notation:

$$
\begin{aligned}
& C=\left[H,\left(n_{\mathbf{i} \bar{\sigma}}+n_{\mathbf{j} \sigma}\right) \Delta_{\mathbf{i j}}\right] \\
& =\left[\left(H_{\mathrm{kin}}+H_{\mathrm{int}}\right),\left(n_{\mathbf{i} \bar{\sigma}}+n_{\mathbf{j} \sigma}\right) \Delta_{\mathbf{i j}}\right]-2 \mu\left(n_{\mathbf{i} \bar{\sigma}}+n_{\mathbf{j} \sigma}\right) \Delta_{\mathbf{i j}} .
\end{aligned}
$$

Neglecting "double occupancy" terms in the commutator with $H_{\text {kin }}$, we get

$$
\begin{aligned}
& {\left[H_{\mathrm{kin}},\left(n_{\mathbf{i} \bar{\sigma}}+n_{\mathbf{j} \sigma}\right) \Delta_{\mathbf{i} \mathbf{j}}\right] \approx-t \sum_{\mathbf{a}} n_{\mathbf{i} \bar{\sigma}} c_{\mathbf{i}+\mathbf{a} \sigma}^{\dagger} c_{\mathbf{j} \bar{\sigma}}^{\dagger}\left(1-\delta_{\mathbf{j} \mathbf{i}+\mathbf{a}}\right)} \\
& +n_{\mathbf{j} \sigma} c_{\mathbf{i} \sigma}^{\dagger} c_{\mathbf{j}+\mathbf{a} \bar{\sigma}}^{\dagger}\left(1-\delta_{\mathbf{j}+\mathbf{a} \mathbf{i}}\right)+c_{\mathbf{i}+\mathbf{a} \bar{\sigma}}^{\dagger} c_{\mathbf{i} \bar{\sigma}} c_{\mathbf{i} \sigma}^{\dagger} c_{\mathbf{j} \bar{\sigma}}^{\dagger}\left(1-\delta_{\mathbf{j} \mathbf{i}+\mathbf{a}}\right) \\
& +c_{\mathbf{j}+\mathbf{a} \sigma}^{\dagger} c_{\mathbf{j} \sigma} c_{\mathbf{i} \sigma}^{\dagger} c_{\mathbf{j} \bar{\sigma}}^{\dagger}\left(1-\delta_{\mathbf{j}+\mathbf{a i}}\right) .
\end{aligned}
$$

The factors of the type $1-\delta_{\mathbf{i j}}$ appear for the following reason. Consider:

$$
\begin{aligned}
n_{\mathbf{j} \sigma} c_{\mathbf{i} \sigma}^{\dagger} c_{\mathbf{j}+\mathbf{a} \bar{\sigma}}^{\dagger} & =n_{\mathbf{j} \sigma} c_{\mathbf{i} \sigma}^{\dagger} c_{\mathbf{j}+\mathbf{a} \bar{\sigma}}^{\dagger}\left(1-\delta_{\mathbf{i j}+\mathbf{a}}\right) \\
& -n_{\mathbf{j} \sigma} c_{\mathbf{i} \sigma}^{\dagger} c_{\mathbf{j}+\mathbf{a} \bar{\sigma}}^{\dagger} \delta_{\mathbf{i j}+\mathbf{a} .}
\end{aligned}
$$

The last term corresponds to the case of two electrons at the same site $\mathbf{i}=\mathbf{j}+\mathbf{a}$. It is small and can be omitted. Applying the Hubbard-I decoupling and taking into account that in the absence of the magnetization $\left\langle c_{\mathbf{i} \sigma} c_{\mathbf{i} \bar{\sigma}}^{\dagger}\right\rangle=0$, we obtain

$$
\begin{aligned}
& {\left[H_{\mathrm{kin}},\left(n_{\mathbf{i} \bar{\sigma}}+n_{\mathbf{j} \sigma}\right) \Delta_{\mathbf{i} \mathbf{j}}\right] \approx-t \sum_{\mathbf{a}}\left\langle n_{\mathbf{i} \bar{\sigma}}\right\rangle c_{\mathbf{i}+\mathbf{a} \sigma}^{\dagger} c_{\mathbf{j} \bar{\sigma}}^{\dagger}\left(1-\delta_{\mathbf{j} \mathbf{i}+\mathbf{a}}\right)} \\
& +\left\langle n_{\mathbf{j} \sigma}\right\rangle c_{\mathbf{i} \sigma}^{\dagger} c_{\mathbf{j}+\mathbf{a} \bar{\sigma}}^{\dagger}\left(1-\delta_{\mathbf{j}+\mathbf{a i}}\right) .
\end{aligned}
$$

Then, we calculate the commutator with $H_{\text {int }}$ :

$$
\begin{aligned}
& {\left[H_{\mathrm{int}},\left(n_{\mathbf{i} \bar{\sigma}}+n_{\mathbf{j} \sigma}\right) \Delta_{\mathbf{i j}}\right]=\left(n_{\mathbf{i} \bar{\sigma}}+n_{\mathbf{j} \sigma}\right)\left[H_{\mathrm{int}}, \Delta_{\mathbf{i j}}\right]} \\
& =U\left(n_{\mathbf{i} \bar{\sigma}}+n_{\mathbf{j} \sigma}\right)^{2} \Delta_{\mathbf{i j}} .
\end{aligned}
$$

We note that $n_{\mathbf{j} \sigma}^{2}=n_{\mathbf{j} \sigma}$ and $U n_{\mathbf{i} \bar{\sigma}} n_{\mathbf{j} \sigma} \Delta_{\mathbf{i j}}=O(t / U)$. As a result, it holds:

$$
\left[H_{\mathrm{int}},\left(n_{\mathbf{i} \bar{\sigma}}+n_{\mathbf{j} \sigma}\right) \Delta_{\mathbf{i} \mathbf{j}}\right] \approx U\left(n_{\mathbf{i} \bar{\sigma}}+n_{\mathbf{j} \sigma}\right) \Delta_{\mathbf{i j}} .
$$

Collecting all terms together, we derive

$$
\begin{aligned}
& {\left[H,\left(n_{\mathbf{i} \bar{\sigma}}+n_{\mathbf{j} \sigma}\right) \Delta_{\mathbf{i} \mathbf{j}}\right] \approx-t\left\langle n_{\sigma}\right\rangle \sum_{\mathbf{a}} c_{\mathbf{i}+\mathbf{a} \sigma}^{\dagger} c_{\mathbf{j} \bar{\sigma}}^{\dagger}\left(1-\delta_{\mathbf{j} \mathbf{i}+\mathbf{a}}\right)} \\
& +c_{\mathbf{i} \sigma}^{\dagger} c_{\mathbf{j}+\mathbf{a} \bar{\sigma}}^{\dagger}\left(1-\delta_{\mathbf{j}+\mathbf{a i}}\right)+U\left(n_{\mathbf{i} \bar{\sigma}}+n_{\mathbf{j} \sigma}\right) \Delta_{\mathbf{i j}}
\end{aligned}
$$

where we denote $\left\langle n_{\mathbf{i} \sigma}\right\rangle=\left\langle n_{\mathbf{j} \bar{\sigma}}\right\rangle=\left\langle n_{\sigma}\right\rangle$. 
Two-particle propagator with the help of Hubbard-I approximation

Finally, we calculate the commutator:

$$
\begin{aligned}
& {\left[\left(n_{\mathbf{i} \bar{\sigma}}+n_{\mathbf{j} \sigma}\right) \Delta_{\mathbf{i j}}, \Delta_{\mathbf{l m}}^{\dagger}\right]=\left(n_{\mathbf{j} \sigma} c_{\mathbf{i} \sigma}^{\dagger} c_{\mathbf{j} \bar{\sigma}}+n_{\mathbf{i} \bar{\sigma}} c_{\mathbf{i} \sigma}^{\dagger} c_{\mathbf{l} \sigma}\right) \delta_{\mathbf{j} \mathbf{m}}} \\
& -\left(n_{\mathbf{i} \bar{\sigma}} c_{\mathbf{m} \bar{\sigma}} c_{\mathbf{j} \bar{\sigma}}^{\dagger}+n_{\mathbf{j} \sigma} c_{\mathbf{m} \bar{\sigma}} c_{\mathbf{j} \bar{\sigma}}^{\dagger}\right) \delta_{\mathbf{i l}} \\
& -\left(c_{\mathbf{m} \bar{\sigma}} c_{\mathbf{m} \sigma}^{\dagger} c_{\mathbf{l} \sigma} c_{\mathbf{j} \bar{\sigma}}^{\dagger} \delta_{\mathbf{i} \mathbf{m}}+c_{\mathbf{m} \bar{\sigma}} c_{\mathbf{i} \sigma}^{\dagger} c_{\mathbf{l} \sigma} c_{\mathbf{j} \bar{\sigma}}^{\dagger} \delta_{\mathbf{l j}}\right) .
\end{aligned}
$$

Deriving the latter formula one has to keep in mind that $\delta_{\mathbf{j} \mathbf{m}} \delta_{\mathbf{j} \mathbf{1}}=\delta_{\mathbf{j m}} \delta_{\mathbf{l m}}=0$, when $\mathbf{i} \neq \mathbf{j}$ and $\mathbf{l} \neq \mathbf{m}$. From $(\underline{A .9}$ ) it is possible to deduce:

$$
\begin{aligned}
\left\langle\left[\left(n_{\mathbf{i} \bar{\sigma}}+n_{\mathbf{j} \sigma}\right) \Delta_{\mathbf{i j}}, \Delta_{\mathbf{l m}}^{\dagger}\right]\right\rangle & \approx\left\langle n_{\mathbf{j} \sigma}\right\rangle\left\langle c_{\mathbf{i} \sigma}^{\dagger} c_{\mathbf{l} \sigma}\right\rangle \delta_{\mathbf{j} \mathbf{m}} \\
& -\left\langle n_{\mathbf{i} \bar{\sigma}}\right\rangle\left\langle c_{\mathbf{m} \bar{\sigma}} c_{\mathbf{j} \bar{\sigma}}^{\dagger}\right\rangle \delta_{\mathbf{i l}},
\end{aligned}
$$

Obtaining this result we took into account that in the absence of the magnetic order

$$
\left\langle c_{\mathbf{m} \bar{\sigma}} c_{\mathbf{m} \sigma}^{\dagger}\right\rangle=\left\langle c_{\mathbf{j} \sigma} c_{\mathbf{j} \bar{\sigma}}^{\dagger}\right\rangle=0 .
$$

Further, in (A.10) the terms

$$
n_{\mathbf{i} \bar{\sigma}} c_{\mathbf{i} \sigma}^{\dagger} c_{\mathbf{l} \sigma} \delta_{\mathbf{j} \mathbf{m}}=O(t / U), \quad c_{\mathbf{m} \bar{\sigma}} n_{\mathbf{j} \sigma} c_{\mathbf{j} \bar{\sigma}}^{\dagger}=O(t / U),
$$

are dropped. Besides, we performed Hubbard-I decoupling in three-site operators:

$$
\begin{aligned}
& n_{\mathbf{j} \sigma} c_{\mathbf{i} \sigma}^{\dagger} c_{\mathbf{l} \sigma} \approx\left\langle n_{\mathbf{j} \sigma}\right\rangle c_{\mathbf{i} \sigma}^{\dagger} c_{\mathbf{l} \sigma}\left(1-\delta_{\mathbf{j} \mathbf{1}}\right), \\
& c_{\mathbf{m} \bar{\sigma}} c_{\mathbf{m} \sigma}^{\dagger} c_{\mathbf{l} \sigma} c_{\mathbf{j} \bar{\sigma}}^{\dagger} \approx\left\langle c_{\mathbf{m} \bar{\sigma}} c_{\mathbf{m} \sigma}^{\dagger}\right\rangle c_{\mathbf{l} \sigma} c_{\mathbf{j} \bar{\sigma}}^{\dagger}=0 .
\end{aligned}
$$

In A.13) we used the fact that $n_{\mathbf{j} \sigma} c_{\mathbf{l} \sigma} \delta_{\mathbf{j} \mathbf{1}}=0$. As a result, within the scope of our approximation, equation (A.1) can be written as

$$
\begin{aligned}
& \dot{\mathcal{P}}_{\mathbf{i j l m}} \approx-t\left\langle n_{\sigma}\right\rangle \sum_{\mathbf{a}}\left[P_{\mathbf{i}+\mathbf{a j l m}}\left(1-\delta_{\mathbf{j} \mathbf{i}+\mathbf{a}}\right)+P_{\mathbf{i} \mathbf{j}+\mathbf{a l m}}\left(1-\delta_{\mathbf{j}+\mathbf{a i}}\right)\right] \\
& +(U-2 \mu) \mathcal{P}_{\mathbf{i j l m}}+\delta(\tau)\left\langle n_{\sigma}\right\rangle\left(\left\langle c_{\mathbf{i} \sigma}^{\dagger} c_{\mathbf{l} \sigma}\right\rangle \delta_{\mathbf{j} \mathbf{m}}-\left\langle c_{\mathbf{m} \bar{\sigma} \overline{\mathbf{j} \sigma}} c^{\dagger}\right\rangle \delta_{\mathbf{i l}}\right) .
\end{aligned}
$$

Since $\mathcal{P}=O(t / U)$, while $P=O(1)$, we omit $\dot{\mathcal{P}}$ and $\mu \mathcal{P}$ terms, arriving at equation (19).

\section{Appendix B. Fourier transformation}

To solve (21), analytically or numerically, it is useful to subject this equation to the Fourier transformation. Let us consider a square 2D lattice with the number of sites $N=M \times M$. In Fourier space equation (21) reads:

$$
P_{\mathbf{k}_{1} \mathbf{k}_{2} \mathbf{k}_{3} \mathbf{k}_{4} \omega}\left[i \omega+\tilde{t} \sum_{\mathbf{a}}\left(e^{i \mathbf{k}_{1} \mathbf{a}}+e^{i \mathbf{k}_{2} \mathbf{a}}\right)+2 \mu\right]=\mathcal{S}+\mathcal{R} .
$$

Here

$$
\begin{aligned}
\mathcal{S} & =\frac{\tilde{t}}{N^{2}} \sum_{\mathbf{a} \mathbf{i} \mathbf{j}} e^{i \mathbf{k}_{1} \mathbf{i}+i \mathbf{k}_{2} \mathbf{j}}\left[\left(\delta_{\mathbf{i}+\mathbf{a} \mathbf{j}}+\delta_{\mathbf{i} \mathbf{j}}\right) P_{\mathbf{i}+\mathbf{a} \mathbf{j} \mathbf{k}_{3} \mathbf{k}_{4} \omega}\right. \\
& \left.+\left(\delta_{\mathbf{i} \mathbf{j}+\mathbf{a}}+\delta_{\mathbf{i} \mathbf{j}}\right) P_{\mathbf{i} \mathbf{j}+\mathbf{a} \mathbf{k}_{3} \mathbf{k}_{4} \omega}\right], \\
\mathcal{R} & =\frac{1-\left\langle n_{\mathbf{i} \sigma}\right\rangle}{N^{2}}\left(\left\langle c_{\mathbf{k}_{1} \sigma}^{\dagger} c_{\mathbf{k}_{1} \sigma}\right\rangle-\left\langle c_{\mathbf{k}_{2} \bar{\sigma}} c_{\mathbf{k}_{2} \bar{\sigma}}^{\dagger}\right\rangle\right) \delta_{\mathbf{k}_{1} \mathbf{k}_{3}} \delta_{\mathbf{k}_{2} \mathbf{k}_{4}} .
\end{aligned}
$$


After straightforward algebra we derive

$$
\mathcal{S}=\frac{z \tilde{t}}{N} \sum_{\mathbf{q}}\left(\gamma_{\mathbf{k}_{1}}+\gamma_{\mathbf{k}_{2}}+\gamma_{\mathbf{k}_{1}-\mathbf{q}}+\gamma_{\mathbf{k}_{2}+\mathbf{q}}\right) P_{\mathbf{k}_{1}-\mathbf{q}, \mathbf{k}_{2}+\mathbf{q}, \mathbf{k}_{3}, \mathbf{k}_{4}, \omega}
$$

where the number of the nearest neighbors on the lattice $z=4$, and we introduce the notation

$$
\gamma_{\mathbf{k}}=\frac{1}{z} \sum_{\mathbf{a}} \exp (i \mathbf{k a})
$$

The term $\mathcal{R}$ in the right-hand side of equation (B.1) is transformed with the help of the formula for the single-electron Green's function in the Hubbard-I approximation:

$$
\left\langle c_{\mathbf{i} \sigma} c_{\mathbf{j} \sigma}^{\dagger}\right\rangle=-\left(1-\left\langle n_{\bar{\sigma}}\right\rangle\right) G_{0}(\mathbf{i}-\mathbf{j},+0),
$$

where $G_{0}$ is the Green's function for free fermions. From this equation it follows that:

$$
\left\langle c_{\mathbf{k} \sigma} c_{\mathbf{k} \sigma}^{\dagger}\right\rangle=\left(1-\left\langle n_{\bar{\sigma}}\right\rangle\right)\left[1-n_{\mathrm{F}}\left(\tilde{\varepsilon}_{\mathbf{k}}-\mu\right)\right],
$$

where $n_{\mathrm{F}}$ is Fermi distribution function, and $\tilde{\varepsilon}_{\mathbf{k}}$ is the energy spectrum. In the considered case of the square lattice and strong electron-electron repulsion $(U \gg z t)$ we can write in the tight-binding approximation: $\tilde{\varepsilon}_{\mathbf{k}}=z \tilde{t} \gamma_{\mathbf{k}}$.

Within the Hubbard-I approach expectation value $\left\langle c_{\mathbf{j} \sigma}^{\dagger} c_{\mathbf{i} \sigma}\right\rangle$ satisfies:

$$
\left\langle c_{\mathbf{j} \sigma}^{\dagger} c_{\mathbf{i} \sigma}\right\rangle=-\left\langle c_{\mathbf{i} \sigma} c_{\mathbf{j} \sigma}^{\dagger}\right\rangle+\left(1-\left\langle n_{\bar{\sigma}}\right\rangle\right) \delta_{\mathbf{i j}} .
$$

This expression is incompatible with the usual Fermi anticommutation rules. This is an artifact of the Hubbard-I scheme, a discrepancy which is a consequence of the inexact nature of the Hubbard-I Green's functions. This means that the Hubbard-I Green's function fails at energy higher than $\tilde{t}$, or, equivalently, at $|\mathbf{i}-\mathbf{j}|$ smaller than at least several lattice constants.

Using the above relations we derive after simple algebra

$$
\mathcal{R}=\frac{\left(1-\left\langle n_{\sigma}\right\rangle\right)^{2}}{M^{2}}\left[n_{\mathrm{F}}\left(\tilde{\varepsilon}_{\mathbf{k}_{1}}-\mu\right)+n_{\mathrm{F}}\left(\tilde{\varepsilon}_{\mathbf{k}_{2}}-\mu\right)-1\right] \delta_{\mathbf{k}_{1} \mathbf{k}_{3}} \delta_{\mathbf{k}_{2} \mathbf{k}_{4}} .
$$

Combining the expressions for $\mathcal{S}$ and $\mathcal{R}$, we can rewrite (B.1) in the form

$$
\begin{aligned}
& {\left[i \omega+z \tilde{t}\left(\gamma_{\mathbf{k}_{1}}+\gamma_{\mathbf{k}_{2}}\right)+2 \mu\right] P_{\mathbf{k}_{1} \mathbf{k}_{2} \mathbf{k}_{3} \mathbf{k}_{4} \omega}} \\
& =\frac{z \tilde{t}}{M} \sum_{\mathbf{q}}\left(\gamma_{\mathbf{k}_{1}}+\gamma_{\mathbf{k}_{2}}+\gamma_{\mathbf{k}_{1}-\mathbf{q}}+\gamma_{\mathbf{k}_{2}-\mathbf{q}}\right) P_{\mathbf{k}_{1}-\mathbf{q}, \mathbf{k}_{2}+\mathbf{q}, \mathbf{k}_{3}, \mathbf{k}_{4}, \omega}+ \\
& \frac{\left(1-\left\langle n_{\sigma}\right\rangle\right)^{2}}{M^{2}}\left[n_{\mathrm{F}}\left(\tilde{\varepsilon}_{\mathbf{k}_{1}}-\mu\right)+n_{\mathrm{F}}\left(\tilde{\varepsilon}_{\mathbf{k}_{2}}-\mu\right)-1\right] \delta_{\mathbf{k}_{1} \mathbf{k}_{3}} \delta_{\mathbf{k}_{2} \mathbf{k}_{4}} .
\end{aligned}
$$

The propagator $P_{\mathbf{k}_{1} \mathbf{k}_{2} \mathbf{k}_{3} \mathbf{k}_{4} \omega}$ is non-zero only if $\mathbf{k}_{1}+\mathbf{k}_{2}=\mathbf{k}_{3}+\mathbf{k}_{4}$. This is a consequence of the momentum conservation law. Therefore, it is convenient to introduce the total momentum $\mathbf{k}_{1}+\mathbf{k}_{2}=\mathbf{Q}$ and define

$$
P_{\mathbf{k}_{i} \mathbf{k}_{f} \omega}^{\mathbf{Q}}=P_{\mathbf{k}_{i}, \mathbf{Q}-\mathbf{k}_{i}, \mathbf{k}_{f}, \mathbf{Q}-\mathbf{k}_{f}, \omega} .
$$

Equation (B.8) can be rewritten as

$$
i \omega P_{\mathbf{k}_{i} \mathbf{k}_{f} \omega}^{\mathbf{Q}}=\sum_{\mathbf{q}} h_{\mathbf{k}_{i} \mathbf{q}}^{\mathbf{Q}} P_{\mathbf{q k}_{f} \omega}^{\mathbf{Q}}+\mathcal{R}_{\mathbf{k}_{i} \mathbf{k}_{f}}^{\mathbf{Q}},
$$


where

$$
\begin{aligned}
h_{\mathbf{k}_{i} \mathbf{q}}^{\mathbf{Q}} & =-\left[z \tilde{t}\left(\gamma_{\mathbf{k}_{i}}+\gamma_{\mathbf{Q}-\mathbf{k}_{i}}\right)+2 \mu\right] \delta_{\mathbf{q k}_{i}} \\
& +\frac{z \tilde{t}}{M}\left(\gamma_{\mathbf{k}_{i}}+\gamma_{\mathbf{Q}-\mathbf{k}_{i}}+\gamma_{\mathbf{q}}+\gamma_{\mathbf{Q}-\mathbf{q}}\right), \\
\mathcal{R}_{\mathbf{k}_{i} \mathbf{k}_{f}}^{\mathbf{Q}} & =\frac{\left(1-\left\langle n_{\sigma}\right\rangle\right)^{2}}{M^{2}} \\
& \times\left[n_{\mathrm{F}}\left(\tilde{\varepsilon}_{\mathbf{k}_{i}}-\mu\right)+n_{\mathrm{F}}\left(\tilde{\varepsilon}_{\mathbf{Q}-\mathbf{k}_{i}}-\mu\right)-1\right] \delta_{\mathbf{k}_{i} \mathbf{k}_{f}} .
\end{aligned}
$$

Equation (B.10) may be used to calculate the propagator $P$.

\section{Appendix C. Computation of the equal-time correlation function}

Equation (B.10) may be cast in the matrix form $i \omega \mathbf{P}=\mathbf{h P}+\mathcal{R}$. Matrix $\mathbf{h}$ is symmetric and, consequently, can be diagonalized. We write down $\mathbf{h}$ symbolically as $\mathbf{h}=\mathbf{U d U}^{+}$, where $\mathbf{d}$ is a diagonal matrix with the diagonal elements $d_{i}$ :

$$
\mathbf{d}=\operatorname{diag}\left(d_{i}\right),
$$

$\mathbf{U}$ is a unitary matrix, $\mathbf{U}^{+}$is its Hermitian-conjugated matrix. Thus, the solution of the problem can be expressed in the form

$$
\mathbf{P}(\omega)=\left[\mathbf{U}(i \omega-\mathbf{d}) \mathbf{U}^{+}\right]^{-1} \mathcal{R} .
$$

We diagonalize numerically the matrix $\mathbf{h}$ and calculate the equal-time propagator $P_{\mathrm{ijlm}}(+0)=\left\langle\Delta_{\mathrm{ij}} \Delta_{\mathrm{lm}}^{\dagger}\right\rangle$ performing frequency summation

$$
\mathbf{P}(\tau \rightarrow+0)=\left.T \sum_{\omega} \exp (i \tau \omega) \mathbf{P}(\omega)\right|_{\tau \rightarrow+0}
$$

according the rule

$$
\left.T \sum_{\omega} \exp (i \tau \omega)(i \omega-\mathbf{d})^{-1}\right|_{\tau \rightarrow+0}=N_{\mathrm{B}}(\mathbf{d})
$$

where $N_{\mathrm{B}}(\varepsilon)=\left(e^{\varepsilon / T}-1\right)^{-1}$ is Bose distribution function, and $N_{\mathrm{B}}(\mathbf{d})$ is a diagonal matrix: $N_{\mathrm{B}}(\mathbf{d})=\operatorname{diag}\left[N_{\mathrm{B}}\left(d_{i}\right)\right]$. As a result, we derive

$$
\mathbf{P}(\tau \rightarrow 0)=\mathbf{U} N_{\mathrm{B}}(\mathbf{d}) \mathbf{U}^{+} \mathcal{R} .
$$

This equation can be used to calculate the correlation function numerically.

\section{References}

[1] Hubbard J 1963 Proc. Roy. Soc. A (London) 276238

[2] Kohn W and Luttinger J M (1965) Phys. Rev. Lett. 15524

[3] Scalapino D J, Loh E Jr, and Hirsch J E 1986 Phys. Rev. B 348190

[4] Koikegami S and Yanagisawa T 2006 J. Phys. Soc. Jpn. 75034715

[5] Yanagisawa T, 2008 New J. Phys. 10023014

[6] Baranov M A and Kagan M Yu 1992 Z.Phys. B-Condens. Matter 86237

[7] Baranov M A, Chubukov A V and Kagan M Yu 1992 Int. J. Mod. Phys. B 62471

[8] Kagan M Yu, Fresard R, Capezzali M and Beck H 1998 Phys. Rev. B 575995 
[9] Zhang S, Carlson J and Gubernatis J E 1997 Phys. Rev. Lett. 784486

[10] Aimi T and Imada M 2007 J. Phys. Soc. Jpn. 76113708

[11] Yokoyama H, Tanaka Y, Ogata M and Tsuchiura H 2004 J. Phys. Soc. Jpn. 73 1119; Maier T A, Jarrell M, Schulthess T C, Kent P R C and White J B 2005 Phys. Rev. Lett. 95 237001; Sénéchal D, Lavertu P-L, Marois M-A and Tremblay A-M S 2005 Phys. Rev. Lett. 94 156404; Capone M and Kotliar G 2006 Phys. Rev. B 74 054513; Aichhorn M, Arrigoni E, Potthoff M and Hanke W 2006 Phys. Rev. B 74 024508; Yokoyama H, Ogata M and Tanaka Y 2006 J. Phys. Soc. Jpn. 75, 114706; Baeriswyl D, Eichenberger D and Menteshashvili M 2009 New J. Phys. 11075010

[12] Domański T and Wysokiński K I 1999 Phys. Rev. B 59173

[13] Mierzejewski M 1996 J. of Supercond. 9293

[14] Zieliński J, Mierzejewski M and Entel P 1998 Phys. Rev. B 5710311

[15] Mierzejewski M, Zieliński J and Entel P 1998 Acta Phys. Pol. B 293907

[16] Cebula A and Zieliński J 1999 J. of Supercond. 12649

[17] Caixeiro E S and Troper A 2008 Physica B 4031071

[18] Caixeiro E S and Troper A 2008 J. Magn. Magn. Mater. 320 e490

[19] Beenen J and Edwards D M 1995 Phys. Rev. B 5213636

[20] Calegari E J, Magalhães S G and Gomes A A 2005 EPJB 45485

[21] Plakida N M 1998 Cond. Mat. Phys. 157

[22] Ovchinnikov S G and Val'kov V V 2004 Hubbard Operators in the Theory of Strongly Correlated Electron, (London: Imperial College Press)

[23] Zaitsev R O 2007 Diagrammatic Methods in the Theory of Superconductivity and Ferromagnetism (Moscow: URSS)

[24] Corney J F and Drummond P D 2006 Phys. Rev. B 73 125112; Corney J F and Drummond P D 2006 J. Phys. A: Math. Gen. 39 269; Aimi T and Imada M 2007 J. Phys. Soc. Jpn. 76084709 